INTERNATIONAL JOURNAL OF ROBUST AND NONLINEAR CONTROL

Int. J. Robust Nonlinear Control 2008; 18:1657-1680

Published online 27 March 2008 in Wiley InterScience (www.interscience.wiley.com). DOI: 10.1002/rnc.1313

\title{
Robust fault estimation of uncertain systems using an LMI-based approach
}

\author{
Euripedes G. Nobrega ${ }^{1}$, Musa O. Abdalla ${ }^{2, *, \dagger}$ and Karolos M. Grigoriadis ${ }^{3}$ \\ ${ }^{1}$ Departamento de Mecanica Computacional, Universidade Estadual de Campinas, Campinas, SP, Brazil \\ ${ }^{2}$ Mechanical Engineering Department, University of Jordan, Amman, Jordan \\ ${ }^{3}$ Mechanical Engineering Department, University of Houston, Houston, U.S.A.
}

\begin{abstract}
SUMMARY
General recent techniques in fault detection and isolation (FDI) are based on $H_{\infty}$ optimization methods to address the issue of robustness in the presence of disturbances, uncertainties and modeling errors. Recently developed linear matrix inequality (LMI) optimization methods are currently used to design controllers and filters, which present several advantages over the Riccati equation-based design methods. This article presents an LMI formulation to design full-order and reduced-order robust $H_{\infty}$ FDI filters to estimate the faulty input signals in the presence of uncertainty and model errors. Several cases are examined for nominal and uncertain plants, which consider a weight function for the disturbance and a reference model for the faults. The FDI LMI synthesis conditions are obtained based on the bounded real lemma for the nominal case and on a sufficient extension for the uncertain case. The conditions for the existence of a feasible solution form a convex problem for the full-order filter, which may be solved via recently developed LMI optimization techniques. For the reduced-order FDI filter, the inequalities include a non-convex constraint, and an alternating projections method is presented to address this case. The examples presented in this paper compare the simulated results of a structural model for the nominal and uncertain cases and show that a degree of conservatism exists in the robust fault estimation; however, more reliable solutions are achieved than the nominal design. Copyright (C) 2008 John Wiley \& Sons, Ltd.
\end{abstract}

Received 30 March 2006; Revised 18 January 2008; Accepted 21 January 2008

KEY WORDS: fault detection and isolation; linear matrix inequalities; robust $H_{\infty}$ filtering; linear systems

\section{INTRODUCTION}

The increasing complexity of engineering systems demands higher safety and reliability, leading to the need for fault detection (FD) and fault tolerant control (FTC) methods. Monitoring and

\footnotetext{
*Correspondence to: Musa O. Abdalla, Mechanical Engineering Department, University of Jordan, Amman, Jordan.

†E-mail: admin@mechatronix.us

Contract/grant sponsor: FAPESP
}

Copyright (C) 2008 John Wiley \& Sons, Ltd. 
diagnosing faults in a system is an important objective in itself, but in an FTC system, the output signals from fault detection and isolation (FDI) modules may also be used to reconfigure the controller module in order to accommodate the fault. To develop FD methods, much effort has been dedicated since the pioneer works of Beard [1] and Jones [2], but the number of real FTC or FDI applications is very low till today. The functions of monitoring and diagnosing systems are necessary to provide early warning of faulty sensors, actuators or system components, avoiding economical loss or dangerous situations for operators or users. Some surveys of the FDI methods [3-6] and books present the basics of the many different methods developed to detect faults $[7,8]$.

Early FDI methods were based on hardware redundancy to reveal malfunctions in sensors or actuators. However, these methods are expensive and their use will be gradually reduced as the analytical methods are becoming more reliable. For critical systems, e.g. aircrafts, satellites, deepspace probes, etc., combinations of both approaches shall reduce the number of redundant hardware components. Analytical redundancy methods for FDI are based on the comparison of the actual measured and expected signals, the so-called residuals, generated through established relations between input and output signals. These methods may be classified into two broad categories, model-based or knowledge-based, which use, respectively, mathematical modeling of the monitored system or some logical description of the measured signals and its components [4].

In general, two separate processes are necessary: residual generation and residual evaluation. The residual signals must be small if no fault is present and must become significant if there is a fault. The first process compares the redundant signals to generate the residuals. The second process aims at evaluating the residual signals in order to achieve three different tasks: detection, isolation and identification of possible faults [3], or, in other words, trigger an alarm when a fault is present, localize it and determine its severity. During the first two decades, some aspects of model-based methods have been the main research themes, such as unknown inputs estimation, how to reduce the influence of disturbances on the residuals and how to maximize its sensitivity to faults. The work turned during the 1990s to adapting control methods such as $H_{2}$ and $H_{\infty}$ [9-14], trying to make the residuals sensitive to faults but insensitive to disturbances and solving the problem through Riccati equations. However, only a mathematical nominal model of the system is usually available, not necessarily considering the presence of exogenous disturbance inputs and noise, and also time-varying parameters and non-modeled plant dynamics. The focus has so been addressed to the search of FDI methods applied to dynamic uncertain systems with modeling errors and unknown inputs. The goals of a current FDI method are to make the residuals not only sensitive to faults and insensitive to disturbances but also robust to dynamical uncertainties and uncertain or time-varying parameters. It is obvious, though, that some frequency distribution differences between the exogenous inputs and faults are necessary, in order to be possible to discriminate the unknown input signals from the faults, through processing of the measured outputs and the generated residuals. The present work intends to contribute to the robust design of modelbased FDI filters for systems presenting parameter and dynamic uncertainties, adopting a linear matrix inequality (LMI)-based approach to solve the problem. Some commentaries about recent developments are presented next.

Among the significant new topics that arose in the last years, the $H_{-}$-index is concerned with the performance ratio between unknown disturbance and residual sensitivity. The initial approach [15] considered the relationship between the $H_{-}$index (regarding that it is not a norm) of the transfer function of the fault vector to the residual and the $H_{\infty}$-norm of the transfer function of the disturbance vector to the residual, leading to a mixed index problem. Extending this formulation, including the uncertain external loop, the work of Chen and Patton [13] presents 
a more complete development, with the solution for the observer based on the $\mu$-analysis method. Adopting the same mixed index formulation, using an LMI approach, but not including the uncertain loop, the configuration presented in Rambeaux et al. [16] makes use of extra dynamics through filtering the output of the observer, leading to better sensitivity of the detector.

Some authors adopted parametric uncertain systems using a polytopic representation for the uncertainties. Hamelin and Sauter [17] presented a frequency domain procedure based on intervaltype parameter uncertainties, leading to a polytopic unstructured uncertain model of the system. Also using the frequency domain, Casavola et al. [18] formulated the FDI filter design problem with two objectives for polytopic uncertain LTI systems, not needing to know a nominal plant model. The objectives are to minimize the $H_{\infty}$-norm of the disturbance to residual map and the maximization of the lowest singular value of the residual to faults map over a prescribed frequency range. This renders a non-convex constraint, which may be recast into a convex one, through the linearization of the corresponding feasibility region, resulting in a quasi-LMI problem. The projection lemma and congruence transformations are used to solve the problem. Frisk and Nielsen [19] address the problem of robust residual generation in the presence of parametric uncertainties and deterministic disturbances that influence the process, focusing on the designing dealing with parametric uncertainty in a structured residual framework.

Separating the formulation as different objectives has been another common approach. A mixed $H_{2}$ and $H_{\infty}$ residual generator is proposed by Khosrowjerdi et al. [20], assuming fixed spectral densities for the unknown disturbances treated as an $\mathrm{H}_{2}$ objective and bounded energy for the uncertain input formulated as an $H_{\infty}$ filtering problem. The authors justify their options considering that the $\mathrm{H}_{2}$-norm cannot guarantee robustness, whereas the $H_{\infty}$-norm can reduce sensitivity to faults but provides robustness to the uncertainties. The main advantage is the possibility of adjusting the trade-off between detection performance and noise sensitivity through a single parameter. An FDI filter for uncertain systems with modeling errors and disturbance inputs, using a two observers approach, is presented by Zhong et al. [21], for a sub-divided state space. The first part of the state variable is only affected by plant input and unknown disturbances, and the second part is only affected by faults. A two-objective $H_{\infty}$ respective problem is formulated and solved using an LMI technique.

Recently, presenting a more complete treatment of uncertain systems and also a multi-objective design method, the approach of Henry and Zolghadri [22] imposes sensitivity to faults through an $H_{-}$-index over specified frequency ranges and $H_{\infty}$ performance to enforce robustness to model uncertainty. It also included additional requirements of regional poles assignment for the FD filter, searching to tune the transient response and a decay rate of the residual.

To enforce the frequency behavior of the FDI filter, Frisk and Nielsen [23] introduced the idea of using a reference model in an FDI framework. Considering that the performance of the FDI filter should be devised as a trade-off between robustness to modeling errors and insensitivity to unknown inputs, Zhong et al. [24] reduced the design problem to a standard model-matching one, through a reference system obtained from nominal design and the synthesis of the filter minimizing the $H_{\infty}$-norm of the difference between the reference model and the residual generator output. The unknown input vector includes disturbances, uninterested fault and some norm-bounded unstructured model uncertainties. The authors consider the major difficulty to be the selection of a reference model with physical meaning from the FDI viewpoint.

Another recent approach is to impose directly as the residuals, the estimation of the fault signals themselves, instead of the differences produced in the output signals [25]. This has the advantage of easily isolating the fault and can be accomplished through a slight change in the index, permitting 
the adoption of $H_{\infty}$ methods to design the filters. The problem easily fits in a standard $H_{\infty}$ optimization control setup, and the observer and the output filter are computed in a single step.

Robust estimation of the faulty input signals is the method adopted in the present work. A thorough treatment of robustness involving parametric and dynamic uncertainties through inputoutput signals representation is adopted, in the sense usually found in the robust controller design area. The proposed method may also be used to design an output observer and achieve FD in a more common type of residual. But considering that FD signals may be part of an FTC system, it is reasonable to expect a better performance from the controller if the fault signals are estimated, instead of a combination of signals without specific significance. An LMI solution of the $H_{\infty}$ filter has been published [26,27], and the general robust treatment for $H_{\infty}$ filter design [28], and they were adapted to robust FDI filter design and formulated as a new set of LMIs. A complete LMI-based approach to estimate the input fault vector using $H_{\infty}$ filtering is presented for the continuous-time FDI nominal and robust problems. The proposed formulation allows the development of necessary and sufficient solvability conditions for the fixed-order FDI filter design. The full-order $H_{\infty}$ FDI filter design is characterized in terms of convex LMIs whose solution is parameterized for all admissible filters, for nominal and uncertain plants. The reduced-order $H_{\infty}$ filter design is characterized by LMIs with additional coupling non-convex matrix rank constraints, and for this case, an alternating projections method is presented that may be applied for both the nominal and uncertain cases. Some simulation examples for a structural system are presented to demonstrate the proposed methods.

The notation used in this work is standard. The transpose of a real matrix $A$ is denoted by $A^{\mathrm{T}}$, and the symbols $>, \geqslant(<, \leqslant)$ are used to denote positive (negative) definite and semidefinite matrices. The $H_{\infty}$ norm of a rational transfer function $F(s)$ is defined as $\|F(s)\|_{\infty}=\max _{\omega} \bar{\sigma}(F(\mathrm{j} \omega))$, where $\bar{\sigma}(\cdot)$ denotes the maximum singular value of a matrix. The $L_{2}$ norm of a vector-valued function $f(t)$, is defined as $\|f(t)\|_{L_{2}}=\left\{\int_{0}^{\infty} f^{\mathrm{T}}(t) f(t) \mathrm{d} t\right\}^{1 / 2}$. The induced matrix norm is given as $\|A\|=$ $\bar{\sigma}(A)=\left\{\lambda_{\max }\left(A A^{\mathrm{T}}\right)\right\}^{1 / 2}$. Given a real $n \times m$ matrix $A$ with rank $r$, an orthogonal complement $A^{\perp}$ is defined as the possibly non-unique matrix that satisfies $A^{\perp} A=0$ and $A^{\perp} A^{\perp \mathrm{T}}>0$. Hence, the orthogonal complement may be computed from the singular-value decomposition of a matrix

$$
A=\left[\begin{array}{ll}
U_{1} & U_{2}
\end{array}\right]\left[\begin{array}{cc}
\Sigma_{1} & 0 \\
0 & 0
\end{array}\right]\left[\begin{array}{c}
V_{1}^{\mathrm{T}} \\
V_{2}^{\mathrm{T}}
\end{array}\right]
$$

as $A^{\perp}=T U_{2}^{\mathrm{T}}$ where $T$ is an arbitrary non-singular matrix. Linear fraction transformations (LFT) are used to represent the plants to be monitored. Considering the matrices $F$ and $S=\left[\begin{array}{ll}S_{11} & S_{12} \\ S_{21} & S_{22}\end{array}\right]$, the lower LFT is defined as $F_{l}(S, F)=S_{11}+S_{12} F\left(I-S_{22} F\right)^{-1} S_{21}$, for appropriately dimensioned matrices and admitting that the inverse exists.

\section{NOMINAL PROBLEM FORMULATION}

In this section, the nominal $H_{\infty}$ FDI filtering problem is formulated based on the LFT form. The corresponding LMIs and their solutions for the full-order problem are presented. Also, an algorithm is proposed to solve the reduced-order problem. 


\subsection{LFT modeling}

Consider a system plant $P$ of order $n_{\mathrm{p}}$ with its state-space representation

$$
\begin{aligned}
& \dot{x}_{\mathrm{p}}=A_{\mathrm{p}} x_{\mathrm{p}}+B_{\mathrm{p}} u+E_{\mathrm{p}} d+F_{\mathrm{p}} f \\
& y_{\mathrm{p}}=C_{\mathrm{p}} x_{\mathrm{p}}+D_{\mathrm{p}} u+G_{\mathrm{p}} d+H_{\mathrm{p}} f
\end{aligned}
$$

where $x_{\mathrm{p}}$ is the state vector, $u$ is the control input, $d$ is the disturbance vector, $f$ is the fault vector and $A_{\mathrm{p}}, B_{\mathrm{p}}, C_{\mathrm{p}}, D_{\mathrm{p}}, E_{\mathrm{p}}, F_{\mathrm{p}}, G_{\mathrm{p}}$ and $H_{\mathrm{p}}$ are real matrices of appropriate dimensions. $F_{\mathrm{p}}$ and $H_{\mathrm{p}}$ are distribution matrices that model actuator, component and sensor additive faults. The block diagram depicted in Figure 1 represents the proposed configuration.

In Figure 1, $P$ represents the plant to be monitored and $F$ is the unknown filter that is to be determined. The estimation error is defined as $e=r-f$, where $r$ is the residual-generated vector of the FDI filter $F$. Our objective is to design a filter $F$ such that $r$ provides an estimate of the fault vector $f$. By examining the patterns and properties of vector $r$, FTC or detection and isolation of faults for monitoring purposes can be accomplished.

Based on the above formulation, the proposed $H_{\infty}$ optimal filtering problem is to find an FDI dynamic filter $F$ to minimize the worst-case estimation error energy $\|e\|_{L_{2}}$ over all bounded energy generalized disturbance $w^{\mathrm{T}}=\left[u^{\mathrm{T}} d^{\mathrm{T}} f^{\mathrm{T}}\right]$, that is

$$
\min _{F} \sup _{w \in L_{2}-\{0\}} \frac{\|e\|_{L_{2}}}{\|w\|_{L_{2}}}
$$

Adopting the index in (2) is equivalent to minimizing the $H_{\infty}$ norm of the transfer function $T_{w e}$ between the generalized disturbance input and the error of the fault estimation. The $\gamma$-suboptimal $H_{\infty}$ FDI filtering problem is to find (if exists) a filter such that $\left\|T_{w e}\right\|_{\infty}<\gamma$, where $\gamma$ is a given positive scalar.

The block diagram in Figure 1 can be rearranged as presented in Figure 2. Weighting functions can be used to shape appropriately the frequency content of the input signals for better FD and disturbance rejection and can be easily taken into account in the formulation.

In Figure 3, the resultant LFT representation for the nominal plant is presented. Using these figures and considering the state-space formulation as

$$
\begin{aligned}
\dot{x}_{\mathrm{s}} & =A_{\mathrm{s}} x_{\mathrm{s}}+B_{w} w \\
e & =C_{e} x_{\mathrm{s}}+D_{e w} w+D_{e r} r \\
y & =C_{y} x_{\mathrm{s}}+D_{y w} w
\end{aligned}
$$

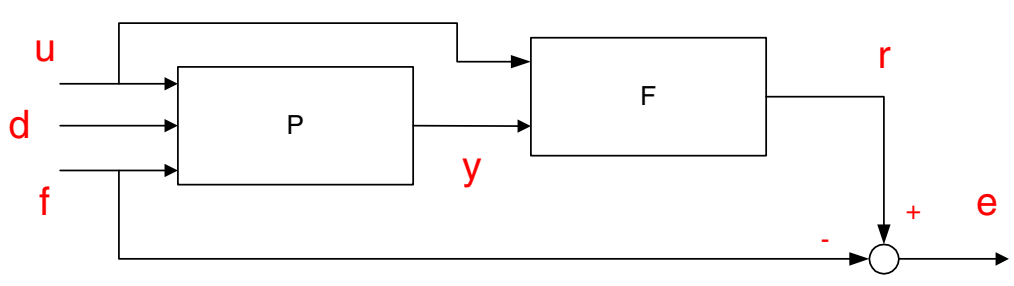

Figure 1. Proposed FDI filter scheme. 


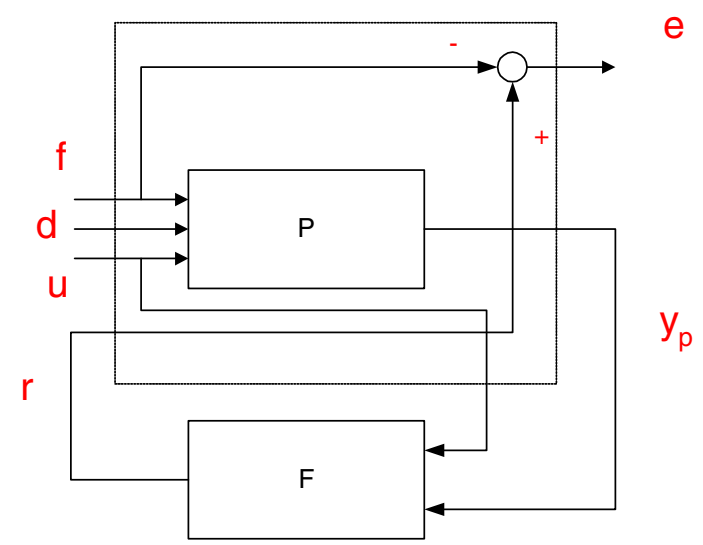

Figure 2. Rearrangement of the FDI configuration.

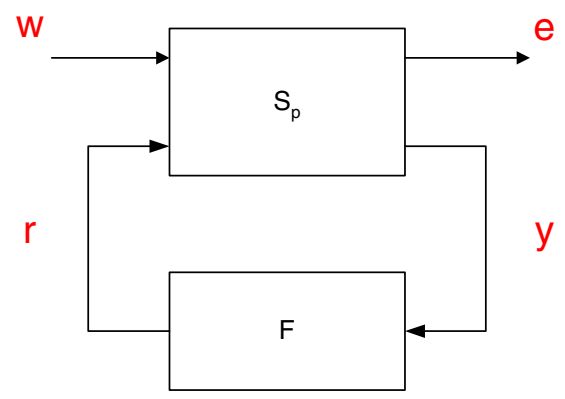

Figure 3. LFT nominal scheme for the filter.

where $w^{\mathrm{T}}=\left[u^{\mathrm{T}} d^{\mathrm{T}} f^{\mathrm{T}}\right]$ represents the combined vector of inputs, control, disturbances and faults and $y^{\mathrm{T}}=\left[u^{\mathrm{T}} y_{\mathrm{p}}^{\mathrm{T}}\right]$ is the combined output. The corresponding state-space representation in a matrix form is given by

$$
\begin{aligned}
\dot{x}_{\mathrm{s}} & =A_{\mathrm{p}} x_{\mathrm{s}}+\left[\begin{array}{lll}
B_{\mathrm{p}} & E_{\mathrm{p}} & F_{\mathrm{p}}
\end{array}\right] w \\
e & =\left[\begin{array}{ll}
0_{n f \times n u} & 0_{n f \times n d}-I_{n f}
\end{array}\right] w+I_{n f} r \\
y & =\left[\begin{array}{c}
0_{n u \times n p} \\
C_{\mathrm{p}}
\end{array}\right] x_{\mathrm{s}}+\left[\begin{array}{ccc}
I_{n u} & 0_{n u \times n d} & 0_{n u \times n f} \\
D_{\mathrm{p}} & G_{\mathrm{p}} & H_{\mathrm{p}}
\end{array}\right] w
\end{aligned}
$$

where $C_{e}=0$ and $D_{e r}=I_{n f}$.

Note that in this case the order of the system $n_{\mathrm{s}}$ is equal to the order of the plant $n_{\mathrm{p}}$, because there are no weight functions or a reference model included. 


\subsection{Continuous-time FDI filter design}

To solve the nominal filter design problem using an LMI-based approach in the continuous-time domain, some algebraic results are presented. Consider a stable $n_{\mathrm{s}}$ th-order linear time-invariant system with the following state-space representation:

$$
\begin{aligned}
\dot{x}_{\mathrm{s}} & =A_{\mathrm{s}} x_{\mathrm{s}}+B_{w} w \\
e & =D_{e w} w+r \\
y & =C_{y} x_{\mathrm{s}}+D_{y w} w
\end{aligned}
$$

where $x_{\mathrm{S}}$ is the state vector, $y$ is the output vector, $w$ is the generalized disturbance vector and $A_{\mathrm{s}}, B_{w}, D_{e w}, C_{y}$ and $D_{y w}$ are real matrices of appropriate dimensions. It is our objective to design a stable linear dynamic filter with the following state-space representation:

$$
\begin{gathered}
\dot{x}_{\mathrm{f}}=A_{\mathrm{f}} x_{\mathrm{f}}+B_{\mathrm{f}} y \\
r=C_{\mathrm{f}} x_{\mathrm{f}}+D_{\mathrm{f}} y
\end{gathered}
$$

where the output $r$ is the estimated fault vector. The vector $x_{\mathrm{f}}$ is the filter state vector, and $A_{\mathrm{f}}, B_{\mathrm{f}}, C_{\mathrm{f}}$, and $D_{\mathrm{f}}$ are real matrices of appropriate dimensions to be computed. The order of the filter $n_{\mathrm{f}}$ is restricted to be less than or equal to the order of the system $n_{\mathrm{s}}$.

An LMI-based $H_{\infty}$ approach will be used to find filter $F$. The filter design is based on the bounded real lemma [29], which is presented next.

\section{Lemma 1}

Consider a stable linear time-invariant system with state-space model

$$
\begin{aligned}
& \dot{x}=A_{c} x+B_{c} w \\
& y=C_{c} x+D_{c} w
\end{aligned}
$$

with transfer function $T_{c}(s)=C_{c}\left(s I-A_{c}\right)^{-1} B_{c}+D_{c}$ and let $\gamma$ be a given positive scalar. Then $\left\|T_{c}\right\|_{\infty}<\gamma$ if and only if there exists a matrix $P>0$ that satisfies

$$
\left[\begin{array}{ccc}
P A_{c}+A_{c}^{\mathrm{T}} P & P B_{c} & C_{c}^{\mathrm{T}} \\
B_{c}^{\mathrm{T}} P & -\gamma^{2} I & D_{c}^{\mathrm{T}} \\
C_{c} & D_{c}^{\mathrm{T}} & -I
\end{array}\right]<0
$$

To find the solvability conditions of the LMI problem in inequality (8), the following lemma may be applied [29].

\section{Lemma 2}

Let $\Gamma, \Lambda$ and $\Theta=\Theta^{\mathrm{T}}$ be given matrices. There exists a matrix $F$ to solve the matrix inequality

$$
\Gamma F \Lambda+\Lambda^{\mathrm{T}} F^{\mathrm{T}} \Gamma^{\mathrm{T}}+\Theta<0
$$


if and only if the following conditions are satisfied:

$$
\begin{array}{r}
\Gamma^{\perp} \Theta \Gamma^{\perp \mathrm{T}}<0 \\
\Lambda^{\mathrm{T} \perp} \Theta \Lambda^{\mathrm{T} \perp \mathrm{T}}<0
\end{array}
$$

These two lemmas may be applied for the case of nominal $H_{\infty}$ FDI filtering to provide the necessary and sufficient conditions for the existence of such filters and the parameterization of all solutions. The following theorem gives the solution to the $\gamma$-suboptimal $H_{\infty}$ FDI filtering problem.

\section{Theorem 1}

There exists an $n_{\mathrm{f}}$ th-order filter $F$ to solve the $\gamma$-suboptimal $H_{\infty}$ FDI filtering problem if and only if there exist matrices $X$ and $Y$ with $Y \geqslant X>0$ such that the following conditions are satisfied:

$$
\begin{array}{r}
{\left[\begin{array}{cc}
X A_{\mathrm{s}}+A_{\mathrm{s}}^{\mathrm{T}} X & X B_{w} \\
B_{w}^{\mathrm{T}} X & -\gamma^{2} I
\end{array}\right]<0} \\
{\left[\begin{array}{c}
C_{y}^{\mathrm{T}} \\
D_{y w}^{\mathrm{T}}
\end{array}\right]^{\perp}\left[\begin{array}{cc}
Y A_{\mathrm{s}}+A_{\mathrm{s}}^{\mathrm{T}} Y & Y B_{w} \\
B_{w}^{\mathrm{T}} Y & D_{e w}^{\mathrm{T}} D_{e w}-\gamma^{2} I
\end{array}\right]\left[\begin{array}{c}
C_{y}^{\mathrm{T}} \\
D_{y w}^{\mathrm{T}}
\end{array}\right]^{\perp \mathrm{T}}<0} \\
\operatorname{rank}(X-Y) \leqslant n_{\mathrm{f}}
\end{array}
$$

The proof of this theorem is presented in Appendix A.

For the full-order FDI filter design $\left(n_{\mathrm{f}}=n_{\mathrm{s}}\right)$, the rank constraint represented by inequality (14) is automatically satisfied and it is not necessary to be included in the formulation. However, the plant order increases when a weighting function or a reference model is included, demanding a reduced-order FDI filter design $\left(n_{\mathrm{f}}<n_{\mathrm{s}}\right)$. This is also a common requirement for complex highorder plants. In this case, the rank constraint renders a non-convex problem, demanding alternate methods to solve the system.

To provide a parameterization of all feasible filters, consider the error system $F_{l}\left(S_{\mathrm{p}}, F\right)$ from the LFT scheme of Figure 3. Defining the state-space vector as $x^{\mathrm{T}}=\left[x_{\mathrm{s}}^{\mathrm{T}} x_{\mathrm{f}}^{\mathrm{T}}\right]$, this error system is described by the following state-space equations:

$$
\begin{aligned}
& \dot{x}=\left[\begin{array}{cc}
A_{\mathrm{s}} & 0 \\
B_{\mathrm{f}} C_{y} & A_{\mathrm{f}}
\end{array}\right] x+\left[\begin{array}{c}
B_{w} \\
B_{\mathrm{f}} D_{y w}
\end{array}\right] w \\
& e=\left[\begin{array}{ll}
D_{\mathrm{f}} C_{y} & C_{\mathrm{f}}
\end{array}\right] x+\left[D_{e w}+D_{\mathrm{f}} D_{y w}\right] w
\end{aligned}
$$

This system can be rewritten as

$$
\begin{aligned}
& \dot{x}=\left(A_{0}+B F M\right) x+\left(B_{0}+B F E\right) w \\
& e=\left(C_{0}+H F M\right) x+\left(D_{0}+H F E\right) w
\end{aligned}
$$


for the respective matrices

$$
\begin{aligned}
A_{0} & =\left[\begin{array}{cc}
A_{\mathrm{s}} & 0 \\
0 & 0
\end{array}\right], \quad B_{0}=\left[\begin{array}{c}
B_{w} \\
0
\end{array}\right], \quad C_{0}=0, \quad D_{0}=D_{e w} \\
B & =\left[\begin{array}{ll}
0 & 0 \\
0 & I
\end{array}\right], \quad M=\left[\begin{array}{cc}
C_{y} & 0 \\
0 & I
\end{array}\right], \quad E=\left[\begin{array}{c}
D_{y w} \\
0
\end{array}\right], \quad H=\left[\begin{array}{ll}
I & 0
\end{array}\right]
\end{aligned}
$$

where the unknown filter matrix is defined as

$$
F=\left[\begin{array}{cc}
D_{\mathrm{f}} & C_{\mathrm{f}} \\
B_{\mathrm{f}} & A_{\mathrm{f}}
\end{array}\right]
$$

Applying Lemma 1, the inequality in (8) becomes

$$
\left[\begin{array}{ccc}
P\left(A_{0}+B F M\right)+\left(A_{0}+B F M\right)^{\mathrm{T}} P & P\left(B_{0}+B F E\right) & (H F M)^{\mathrm{T}} \\
\left(B_{0}+B F E\right)^{\mathrm{T}} P & -\gamma^{2} I & \left(D_{0}+H F E\right)^{\mathrm{T}} \\
H F M & D_{0}+H F E & -I
\end{array}\right]<0
$$

Using Lemma 2, it can be easily devised from (9) that

$$
\Gamma=\left[\begin{array}{c}
P B \\
0 \\
H
\end{array}\right], \quad \Lambda^{\mathrm{T}}=\left[\begin{array}{c}
M^{\mathrm{T}} \\
E^{\mathrm{T}} \\
0
\end{array}\right] \quad \text { and } \quad \Theta=\left[\begin{array}{ccc}
P A_{0}+A_{0}^{\mathrm{T}} P & P B_{0} & 0 \\
B_{0}^{\mathrm{T}} P & -\gamma^{2} I & D_{0}^{\mathrm{T}} \\
0 & D_{0} & -I
\end{array}\right]
$$

Considering the definitions in (16), the following result provides a parameterization of all feasible filters based on the solution $(X, Y)$ of the FDI solvability conditions (12)-(14).

\section{Theorem 2}

All the $\gamma$-suboptimal $H_{\infty}$ FDI $n$ th-order filters $F$ that correspond to a feasible matrix pair $(X, Y)$ are given by

$$
F=\left[\begin{array}{ll}
D_{\mathrm{f}} & C_{\mathrm{f}} \\
B_{\mathrm{f}} & A_{\mathrm{f}}
\end{array}\right]=-R^{-1} \Gamma^{\mathrm{T}} \Phi \Lambda^{\mathrm{T}} \Psi+\Omega^{1 / 2} J \Psi^{1 / 2}
$$

where $\Phi, R$ and $J$ are free matrix parameters subject to

$$
\Phi=\left(\Gamma R^{-1} \Gamma^{\mathrm{T}}-\Theta\right)^{-1}>0, \quad R>0, \quad\|J\|<1
$$

$\Omega$ and $\Psi$ defined by

$$
\begin{aligned}
& \Omega=R^{-1}-R^{-1} \Gamma^{\mathrm{T}}\left(\Phi-\Phi \Lambda^{\mathrm{T}} \Psi \Lambda \Phi\right) \Gamma R^{-1} \\
& \Psi=(\Lambda \Phi \Lambda)^{-1}
\end{aligned}
$$

The proof is not presented here but follows the same approach as in Grigoriadis and Watson [26]. Theorem 2 provides a set of solutions that guarantees the $H_{\infty}$ norm bound for the system. The free parameters may be used then to optimize other system properties. 


\subsection{Reduced-order filter design via alternating projections}

In the previous section, the continuous-time full-order FDI filter design has produced convex LMI constraints, represented by inequalities (12) and (13). On the other hand, the reduced-order FDI filter design requires the inclusion of the rank constraint, inequality (14), leading to a non-convex problem. Recently proposed numerical techniques have been used to solve such reduced-order feasibility and optimization problems [30,31].

In this work, alternating projections are used to solve the $\gamma$-suboptimal reduced-order FDI filter problem. Alternating projections were used in the past in statistical estimation and image reconstruction problems. The basic idea behind these techniques is the following: given a family of convex sets, a sequence of alternating orthogonal projections onto these sets converges to a point in the intersection of the family. Only local convergence is guaranteed for non-convex sets with an initial starting point that is in the vicinity of a feasible solution.

The standard alternating orthogonal projection algorithm is summarized in the following result.

\section{Theorem 3}

Let $\left\{C_{1}, C_{2}, \ldots, C_{n}\right\}$ be a family of closed, convex sets in a Hilbert space such that the intersection $C_{1} \cap C_{2} \cap \cdots \cap C_{n}$ is non-empty and define by $P_{i}$ the orthogonal projection operator onto the set $C_{i}$. Then the sequence of alternating projections

$$
\begin{aligned}
& x_{1}=P_{1} x_{0} \\
& x_{2}=P_{2} x_{1} \\
& \vdots \\
& x_{n}=P_{n} x_{n-1} \\
& x_{n+1}=P_{1} x_{n} \\
& x_{n+2}=P_{2} x_{n+1}
\end{aligned}
$$

converges to a point in the intersection $C_{1} \cap C_{2} \cap \cdots \cap C_{n}$ for any initial vector $x_{0}$. If the intersection is empty, the sequence of alternating projections does not converge.

In order to use the alternating projections techniques, one needs to provide explicit expressions for the orthogonal projections onto the LMI constraints. Expressions for these projections have been derived in [30]. The rank constraint, in the reduced-order FDI design, is enforced using the following iterative scheme.

Step 1: Choose an upper and a lower bound for $\gamma$ and initial values for the matrix pair $(X, Y)$.

Step 2: Solve the feasibility problem, excluding all the non-convex constraints (in this case, it is the rank constraint), for $X$ and $Y$.

Step 3: Use the success or the failure of the previous step in conjunction with the bisection method to update the lower and the higher bounds of $\gamma$.

Step 4: If step 2 is a success (feasible), then project on the non-convex constraints.

Step 5: If the difference of two consecutive $\gamma$ 's is greater than a tolerance, go to step 2. Otherwise, the procedure ends. 


\section{ROBUST FDI PROBLEM FORMULATION}

The robust FDI filter problem will follow the same principles as in the nominal case in order to obtain a parameterization of all possible solutions. To present a more general treatment, a reference model is considered for the fault vector and a weighting function for the disturbance input. The weighting function is used to obtain a desired frequency response for the system, i.e. to improve the performance through a low-pass filter behavior attenuating high-frequency noise. This is basically the same role as in a control system design. FD only demands a significant residual, that is the error in the plant output estimation, but for fault estimation the system needs to be invertible, implying the existence of the direct term between the fault input and the plant output. A strictly proper system does not present this feature, and a non-proper weighting function may be used to provide the necessary invertibility. A reference model can be used to shape the frequency response of the filter, but it may also improve the performance index and assure filter stability for non-minimal phase plants. A solution to include a reference model in the nominal filter design is presented in Nobrega et al. [32].

\subsection{LFT modeling with a reference model}

Consider the following linear time-invariant plant $P$ of order $n_{\mathrm{p}}$ with state-space representation

$$
\begin{aligned}
\dot{x}_{\mathrm{p}} & =A_{\mathrm{p}} x_{\mathrm{p}}+B_{\mathrm{p}} u+E_{\mathrm{p}} y_{d}+F_{\mathrm{p}} f+J_{x} v \\
z & =C_{z} x_{\mathrm{p}}+D_{z} u+G_{z} y_{d}+H_{z} f+J_{z} v \\
y_{\mathrm{p}} & =C_{\mathrm{p}} x_{\mathrm{p}}+D_{\mathrm{p}} u+G_{\mathrm{p}} y_{d}+H_{\mathrm{p}} f+J_{y} v
\end{aligned}
$$

where $x_{\mathrm{p}}$ is the state-space vector, $y_{\mathrm{p}}$ is the output vector, $z$ is the uncertainty output, $u$ is the control input vector, $y_{d}$ is the weighted disturbance of input vector $d, f$ is the fault vector and $v$ is the uncertainty input, such that $v=\Delta(t) z$, where $\Delta(t)$ is a block diagonal function matrix, where each block element is a real diagonal matrix or a full complex matrix and subject to $\|\Delta(t)\|_{\infty}<1$ [29]. This encompasses the most general description, including structured and unstructured uncertainties. $A_{\mathrm{p}}, B_{\mathrm{p}}, C_{\mathrm{p}}, D_{\mathrm{p}}, E_{\mathrm{p}}, F_{\mathrm{p}}, G_{\mathrm{p}}, H_{\mathrm{p}}, C_{z}, D_{z}, G_{z}, H_{z}, J_{x}, J_{y}$ and $J_{z}$ represent the distribution matrices of appropriate dimensions. The block diagram depicted in Figure 4 represents the proposed configuration, where $P$ represents the plant to be monitored, $F$ is the unknown filter, $M$ is the reference model, $W_{d}$ is a weight function for the disturbance and $\Delta$ is the feedback relation for the uncertainty. The estimation error is defined as $e=r-m$, where $r$ is the residual-generated vector of the FDI filter $F$ and $m$ is the output of the reference model. The FDI filter must be designed in order to minimize the error in the presence of the control input, disturbances and uncertainty. The design problem is to find a stable filter $F$, such that its output must follow the fault vector filtered through a chosen reference model $M$, when the plant is subjected to fault, control and disturbance inputs, and uncertainty. If the transfer matrix of the reference model is diagonal, the output of the filter is the fault vector estimation. Otherwise, if it is a rectangular matrix, the filter output is a vector of fault combinations, leading to a fault detector, but with the convenience of filtering each fault signal with a different transfer function, represented by the reference model matrix elements.

The robust filtering problem is to find an FDI dynamic filter $F$ to minimize the worst-case estimation error energy $\|e\|_{L_{2}}$ over all bounded energy generalized disturbance input $w^{\mathrm{T}}=\left[u^{\mathrm{T}} d^{\mathrm{T}} f^{\mathrm{T}}\right]$ 


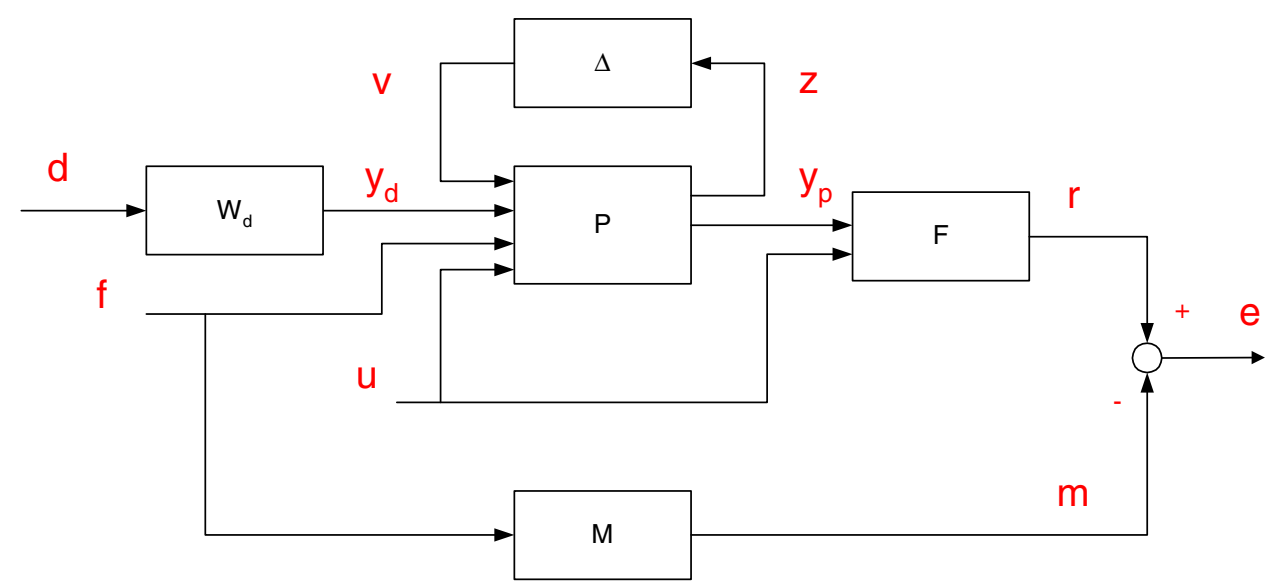

Figure 4. Robust scheme including a reference model and a weight function.

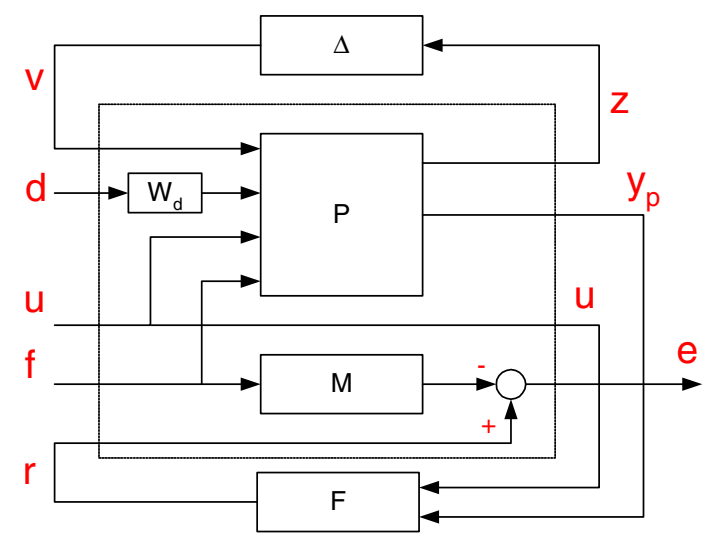

Figure 5. Rearrangement of the block diagram in Figure 4.

despite an uncertainty that is subjected to $\|\Delta(t)\|_{\infty}<1$ constraint. The optimization problem is cast to find a filter that minimizes the following index:

$$
\min _{F} \sup _{\|\Delta\|_{\infty}<1, w \in L_{2}-\{0\}} \frac{\|e\|_{L_{2}}}{\|w\|_{L_{2}}}
$$

Again, adopting the index in (18) is equivalent to minimizing the $H_{\infty}$ norm of the transfer function $T_{w e}$ between the generalized disturbance input and the error of the fault estimation in the presence of uncertainty. The $\gamma$-suboptimal $H_{\infty}$ robust FDI filtering problem is to find (if exists) a filter such that $\sup _{\|\Delta\|_{\infty}<1}\left\|T_{w e}\right\|_{\infty}<\gamma$, where $\gamma$ is a given positive scalar. 
The block diagram shown in Figure 4 is rearranged and represented in Figure 5. To provide good FDI capability, appropriate weighting functions are used to penalize the disturbance, the fault and the uncertainty vectors, in addition to using the reference model. For the sake of simplicity, only the disturbance has been weighted here. The following state-space models represent the weight function and the reference model

$$
\begin{gathered}
\dot{x}_{d}=A_{d} x_{d}+B_{d} d \\
y_{d}=C_{d} x_{d}+D_{d} d \\
\dot{x}_{m}=A_{m} x_{m}+B_{m} f \\
m=C_{m} x_{m}+D_{m} f
\end{gathered}
$$

The rearranged system in Figure 5 is grouped as depicted in Figure 6 in order to use the LFT scheme, where $S_{u}$ represents the plant, reference model and disturbance weighting function all integrated in one block. The state-space formulation for the system $S_{u}$ is

$$
\begin{aligned}
& \dot{x}_{\mathrm{s}}=\left[\begin{array}{ccc}
A_{\mathrm{p}} & E_{\mathrm{p}} C_{d} & 0 \\
0 & A_{d} & 0 \\
0 & 0 & A_{m}
\end{array}\right] x_{\mathrm{s}}+\left[\begin{array}{c}
J_{x} \\
0 \\
0
\end{array}\right] v+\left[\begin{array}{ccc}
B_{\mathrm{p}} & E_{\mathrm{p}} D_{d} & F_{\mathrm{p}} \\
0 & B_{d} & 0 \\
0 & 0 & B_{m}
\end{array}\right] w \\
& z=\left[\begin{array}{llll}
C_{z} & G_{z} C_{d} & 0
\end{array}\right] x_{\mathrm{s}}+J_{z} v+\left[\begin{array}{llll}
D_{z} & G_{z} D_{d} & H_{z}
\end{array}\right] w \\
& e=\left[\begin{array}{lll}
0 & 0 & -C_{m}
\end{array}\right] x_{\mathrm{s}}+\left[\begin{array}{lll}
0 & 0 & -D_{m}
\end{array}\right] w+I_{n f} r \\
& y=\left[\begin{array}{ccc}
0 & 0 & 0 \\
C_{\mathrm{p}} & G_{\mathrm{p}} C_{d} & 0
\end{array}\right] x_{\mathrm{s}}+\left[\begin{array}{c}
0 \\
J_{y}
\end{array}\right] v+\left[\begin{array}{ccc}
I_{n u} & 0 & 0 \\
D_{\mathrm{p}} & G_{\mathrm{p}} D_{d} & H_{\mathrm{p}}
\end{array}\right] w \\
& v=\Delta z
\end{aligned}
$$

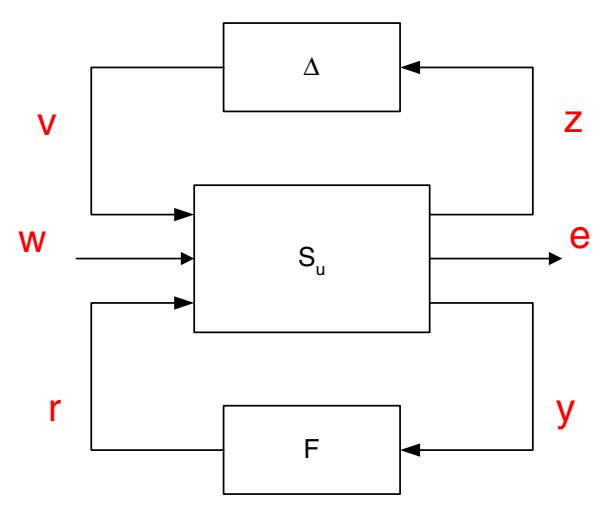

Figure 6. An LFT scheme for the robust filter. 
The following definitions will be adopted for the rest of the paper, where the corresponding matrices are related to (19):

$$
\begin{aligned}
\dot{x}_{\mathrm{s}} & =A_{\mathrm{s}} x_{\mathrm{s}}+B_{v} v+B_{w} w \\
z & =C_{z} x_{\mathrm{s}}+D_{z v} v+D_{z w} w \\
e & =C_{e} x_{\mathrm{s}}+D_{e w} w+I_{n f} r \\
y & =C_{y} x_{\mathrm{s}}+D_{y v} v+D_{y w} w
\end{aligned}
$$

The order of the system $n_{\mathrm{S}}$ is the sum of the order of the plant, the order of the weight function and the order of the reference model. In consequence, the full-order FDI filter will have its order higher than the order of the plant, justifying the need for the reduced-order filter solution.

\subsection{Continuous-time robust FDI filter design}

To solve the robust problem using an LMI-based approach in the continuous-time domain, another lemma and theorem will be presented. Consider a stable $n$ th-order linear time-invariant system represented as in Equation (20) and the linear dynamic filter definition which is given in Equation (6), whose output $r$ is to estimate the fault vector, filtered through the reference model. The order of the filter $n_{\mathrm{f}}$ is again restricted to be less than or equal to the order of the system $S_{u}$.

There is no necessary and sufficient condition for robust stability presently in the literature. The following lemma is a sufficient condition extension for the bounded real lemma, including the uncertainty relation [29]. This sufficient condition makes the solutions tend to be conservative. Introducing a scaling matrix is prone to reduce this conservatism. Defining the scaling matrix $S$ as a block diagonal matrix, where each block is a complex positive-definite full matrix or a real positive diagonal matrix, such that $\Delta=S^{-1 / 2} \Delta S^{1 / 2}$, the lemma may be presented as follows.

\section{Lemma 3}

Consider a stable linear time-invariant uncertain system

$$
\begin{aligned}
& \dot{x}=A_{c} x+B_{c 1} v+B_{c 2} w \\
& z=C_{c z} x+D_{c z 1} v+D_{c z 2} w \\
& e=C_{c e} x+D_{c e 1} v+D_{c e 2} w \\
& v=\Delta z
\end{aligned}
$$

with $\|\Delta(t)\|_{\infty}<1$ and let $\gamma$ be a given positive scalar. Then the closed-loop transfer function condition $\left\|T_{w y}\right\|_{\infty}<\gamma$ holds if there exists a matrix $P>0$ that satisfies

$$
\left[\begin{array}{ccc}
P A_{c}+A_{c}^{\mathrm{T}} P & P B_{c 1} & P B_{c 2} \\
B_{c 1}^{\mathrm{T}} P & -S & 0 \\
B_{c 2}^{\mathrm{T}} P & 0 & -\gamma^{2} I
\end{array}\right]+\left[\begin{array}{cc}
C_{c z}^{\mathrm{T}} & C_{c e}^{\mathrm{T}} \\
D_{c z 1}^{\mathrm{T}} & D_{c e 1}^{\mathrm{T}} \\
D_{c z 2}^{\mathrm{T}} & D_{c e 2}^{\mathrm{T}}
\end{array}\right]\left[\begin{array}{cc}
S & 0 \\
0 & I
\end{array}\right]\left[\begin{array}{ccc}
C_{c z} & D_{c z 1} & D_{c z 2} \\
C_{c e} & D_{c e 1} & D_{c e 2}
\end{array}\right]<0
$$

The solvability condition of Lemma 2 also applies to the inequality of Lemma 3. Using these two lemmas, the sufficient condition for the existence of the robust FDI filter is presented next. The following theorem gives the solution to the $\gamma$-suboptimal robust $H_{\infty}$ FDI filtering problem. 


\section{Theorem 4}

There exists an nth-order filter $F$ to solve the $\gamma$-suboptimal $H_{\infty}$ FDI filtering problem if there exist matrices $X$ and $Y$ with $Y \geqslant X>0$ such that the following conditions are satisfied:

$$
\begin{gathered}
{\left[\begin{array}{cc}
A_{\mathrm{s}} X+X A_{\mathrm{s}}^{\mathrm{T}}+\gamma^{2} B_{v} S^{-1} B_{v}^{\mathrm{T}}+B_{w} B_{w}^{\mathrm{T}} & X C_{z}^{\mathrm{T}}+\gamma^{2} B_{v} S^{-1} D_{z v}^{\mathrm{T}}+B_{w} D_{z w}^{\mathrm{T}} \\
C_{z} X+\gamma^{2} D_{z v} S^{-1} B_{v}^{\mathrm{T}}+D_{z w} B_{w}^{\mathrm{T}} & \gamma^{2}\left(D_{z v} S^{-1} D_{z v}^{\mathrm{T}}-S^{-1}\right)+D_{z w} D_{z w}^{\mathrm{T}}
\end{array}\right]<0} \\
{\left[\begin{array}{c}
C_{y}^{\mathrm{T}} \\
D_{y v}^{\mathrm{T}} \\
D_{y w}^{\mathrm{T}}
\end{array}\right]\left[\begin{array}{ccc}
Y A_{\mathrm{s}}+A_{\mathrm{s}}^{\mathrm{T}} Y+C_{z}^{\mathrm{T}} S C_{z}+C_{e}^{\mathrm{T}} C_{e} & Y B_{v}+C_{z}^{\mathrm{T}} S D_{z v} & Y B_{w}+C_{z}^{\mathrm{T}} S D_{z w}+C_{e}^{\mathrm{T}} D_{e w} \\
B_{v}^{\mathrm{T}} Y+D_{z v}^{\mathrm{T}} S C_{z} & D_{z v}^{\mathrm{T}} S D_{z v}-S & D_{z v}^{\mathrm{T}} S D_{z w} \\
B_{w}^{\mathrm{T}} Y+D_{z w}^{\mathrm{T}} S C_{z}+D_{e w}^{\mathrm{T}} C_{e} & D_{z w}^{\mathrm{T}} S D_{z v} & D_{z w}^{\mathrm{T}} S D_{z w}+D_{e w}^{\mathrm{T}} D_{e w}-\gamma^{2} I
\end{array}\right]} \\
\times\left[\begin{array}{c}
C_{y}^{\mathrm{T}} \\
D_{y v}^{\mathrm{T}} \\
D_{y w}^{\mathrm{T}}
\end{array}\right] \begin{array}{c}
\perp \mathrm{T} \quad \\
<0 \quad\left[\begin{array}{cc}
Y & \gamma I \\
\gamma I & X
\end{array}\right] \geqslant 0 \\
\operatorname{rank}(X-Y) \leqslant n_{\mathrm{f}}
\end{array}
\end{gathered}
$$

The proof of this theorem is presented in Appendix B.

Conditions (23) and (24) of Theorem 3 are convex LMI constraints on the matrix parameters $X$ and $Y$. For the full-order FDI filtering problem, where $n_{\mathrm{f}}=n_{\mathrm{s}}$, the rank constraint (25) is redundant and the computational problem is a convex LMI problem. To find the parameterization of all robust filters, Theorem 2 may be applied again, but it is necessary to find the new matrices. Considering the new state vector as a combination of the plant and filter states, $x^{\mathrm{T}}=\left[x_{\mathrm{s}}^{\mathrm{T}} x_{\mathrm{f}}^{\mathrm{T}}\right]$, the closed-loop system $F_{l}(S, F)$, from the LFT scheme of Figure 6, is described by the following state-space equations:

$$
\begin{aligned}
& \dot{x}=\left[\begin{array}{cc}
A_{\mathrm{s}} & 0 \\
B_{\mathrm{f}} C_{y} & A_{\mathrm{f}}
\end{array}\right] x+\left[\begin{array}{c}
B_{v} \\
B_{\mathrm{f}} D_{y v}
\end{array}\right] v+\left[\begin{array}{c}
B_{w} \\
B_{\mathrm{f}} D_{y w}
\end{array}\right] w \\
& z=\left[\begin{array}{ll}
C_{z} & 0
\end{array}\right] x+D_{z v} v+D_{z w} w \\
& e=\left[\begin{array}{ll}
C_{e}+D_{\mathrm{f}} C_{y} & C_{\mathrm{f}}
\end{array}\right] x+\left(D_{\mathrm{f}} D_{y v}\right) v+\left(D_{e w}+D_{\mathrm{f}} D_{y w}\right) w
\end{aligned}
$$

Adopting the formulation

$$
\begin{aligned}
& \dot{x}=\left(A_{0}+B F M\right) x+\left(B_{01}+B F E_{1}\right) v+\left(B_{02}+B F E_{2}\right) w \\
& z=C_{1} x+D_{011} v+D_{012} w \\
& e=\left(C_{2}+H_{2} F M\right) x+\left(H_{2} F E_{1}\right) v+\left(D_{022}+H_{2} F E_{2}\right) w
\end{aligned}
$$


the new introduced matrices are given by

$$
\begin{aligned}
A_{0} & =\left[\begin{array}{ll}
A_{\mathrm{s}} & 0 \\
0 & 0
\end{array}\right], \quad B_{01}=\left[\begin{array}{c}
B_{v} \\
0
\end{array}\right], \quad B_{02}=\left[\begin{array}{c}
B_{w} \\
0
\end{array}\right] \\
D_{011} & =D_{z v}, \quad D_{012}=D_{z w}, \quad D_{022}=D_{e w} \\
C_{1} & =\left[\begin{array}{ll}
C_{z} & 0
\end{array}\right], \quad C_{2}=\left[\begin{array}{ll}
C_{e} & 0
\end{array}\right] \\
B & =\left[\begin{array}{ll}
0 & 0 \\
0 & I
\end{array}\right], \quad M=\left[\begin{array}{cc}
C_{y} & 0 \\
0 & I
\end{array}\right], \quad E_{1}=\left[\begin{array}{c}
D_{y v} \\
0
\end{array}\right], \quad E_{2}=\left[\begin{array}{c}
D_{y w} \\
0
\end{array}\right], \quad H_{2}=\left[\begin{array}{ll}
I & 0
\end{array}\right]
\end{aligned}
$$

with the unknown filter defined as

$$
F=\left[\begin{array}{ll}
D_{\mathrm{f}} & C_{\mathrm{f}} \\
B_{\mathrm{f}} & A_{\mathrm{f}}
\end{array}\right]
$$

Applying Lemma 3, inequality (22) becomes

$$
\begin{aligned}
& {\left[\begin{array}{ccc}
P(A+B F M)+(A+B F M)^{\mathrm{T}} P & P\left(B_{01}+B F E_{1}\right) & P\left(B_{02}+B F E_{2}\right) \\
\left(B_{01}+B F E_{1}\right)^{\mathrm{T}} P & -S & 0 \\
\left(B_{02}+B F E_{2}\right)^{\mathrm{T}} P & 0 & -\gamma^{2} I
\end{array}\right]} \\
& +\left[\begin{array}{cc}
C_{1}^{\mathrm{T}} & \left(C_{2}+H_{2} F M\right)^{\mathrm{T}} \\
D_{011}^{\mathrm{T}} & \left(H_{2} F E_{1}\right)^{\mathrm{T}} \\
D_{012}^{\mathrm{T}} & \left(D_{022}+H_{2} F E_{2}\right)^{\mathrm{T}}
\end{array}\right]\left[\begin{array}{cc}
S & 0 \\
0 & I
\end{array}\right]\left[\begin{array}{ccc}
C_{1} & D_{011} & D_{012} \\
C_{2}+H_{2} F M & H_{2} F E_{1} & D_{022}+H_{2} F E_{2}
\end{array}\right]<0
\end{aligned}
$$

Applying Schur formula to (27), and using Lemma 2, it can be devised from (9) that

$$
\Gamma=\left[\begin{array}{c}
P B \\
0 \\
0 \\
0 \\
H_{2}
\end{array}\right], \quad \Lambda=\left[\begin{array}{lllll}
M & E_{1} & E_{2} & 0 & 0
\end{array}\right] \text { and } \Theta=\left[\begin{array}{ccccc}
P A+A^{\mathrm{T}} P & P B_{01} & P B_{02} & C_{1}^{\mathrm{T}} & C_{2}^{\mathrm{T}} \\
B_{01}^{\mathrm{T}} P & -S & 0 & D_{011}^{\mathrm{T}} & 0 \\
B_{02}^{\mathrm{T}} P & 0 & -\gamma^{2} I & D_{012}^{\mathrm{T}} & D_{022}^{\mathrm{T}} \\
C_{1} & D_{011} & D_{012} & -S^{-1} & 0 \\
C_{2} & 0 & D_{022} & 0 & -I
\end{array}\right]
$$

Using the new $\Gamma, \Lambda$ and $\Theta$ as defined by (28), Theorem 2 may now be applied to find the parameterization of all robust filters. For the reduced-order problem, the alternating projections method presented in the previous section (nominal case) may be applied. 
Some comments are necessary to explain the use of the scaling matrix in Theorem 4 in the proposed algorithm. This scaling matrix is an attempt to reduce the conservatism of the robust solutions that result from the general representation of the uncertainties and the non-existence of a necessary and sufficient theorem for the robust case. But its inclusion results in a non-convex optimization problem corresponding to inequalities (23)-(25) depending on the representation adopted. Recall that in Theorem 4 the scaling matrix $S$ and also its inverse are present. To address this non-convex problem, an iterative solution is adopted, beginning with an initial value for the scaling matrix and after solving the LMIs of Theorem 4 in terms of $X$ and $Y$ and finding a filter, optimizing the cost $\gamma$ with respect to the $S$ matrix. This procedure is repeated till an acceptable solution is found. The proposed iterative algorithm is guaranteed to converge, but convergence to a global optimum for the $\gamma$ value is not guaranteed. The scaling matrix is similar to constant $D$-scales from complex structured singular value theory [33] and the iterative approach corresponds to the $D-K$ iteration for structured value synthesis [34].

\section{NUMERICAL EXAMPLES}

The proposed method is applied to a simulated example in this section. Consider a second-order mechanical system that consists of a mass, spring and damper system, with parameter uncertainties $\left(\delta_{1}\right.$ and $\left.\delta_{2}\right)$ in the stiffness and damping coefficients, respectively. The system is described by the following state-space equations:

$$
\begin{aligned}
& \dot{x}_{1}=x_{2} \\
& \dot{x}_{2}=-a_{0} x_{1}-a_{1} x_{2}+u+d+f-v_{1}-v_{2} \\
& z_{1}=\alpha_{1} a_{0} x_{1} \\
& z_{2}=\alpha_{2} a_{1} x_{2} \\
& y=\left(b_{0}-b_{2} a_{0}\right) x_{1}+\left(b_{1}-b_{2} a_{1}\right) x_{2}+b_{2} u+b_{2} d+b_{2} f-b_{2} v_{1}-b_{2} v_{2}
\end{aligned}
$$

where $a_{0}=1, a_{1}=1, b_{0}=1, b_{1}=0.3, b_{2}=0.1$. Assume that both the weight function and the reference model are a second-order low-pass filter with two poles at $-3 \mathrm{rad} / \mathrm{s}$, chosen in order to achieve noise rejection without affecting the plant dynamics in the low-frequency range. The uncertainties are $10 \%$ for $\alpha_{1}$ and $40 \%$ for $\alpha_{2}$.

Considering first the plant without weight functions and reference model, the nominal optimal value for the performance index $\gamma$ was found to be 0.7071 and for the robust case 0.8483 . In general, it must be expected that the nominal index should be less than the robust index.

To analyze the performance of these systems, the uncertain model is simulated using the two differently designed filters. The singular value plots of the error transfer functions, i.e. the transfer matrix between the disturbance input and the filtering errors, are presented in Figure 7, for both cases. These graphs were obtained using the nominal and robust filters and the plant being subjected to five pairs of stiffness and damping coefficient uncertainties, respectively $(2,8 \%),(4,16 \%)$, $(6,24 \%),(8,32 \%)$ and $(10,40 \%)$. This scheme of variation in uncertainties is followed in all graphs presented here. For the nominal design, the horizontal line in the bottom corresponds to the $\gamma$ optimal value in decibels. All the plots are above this value, implying that the performance 

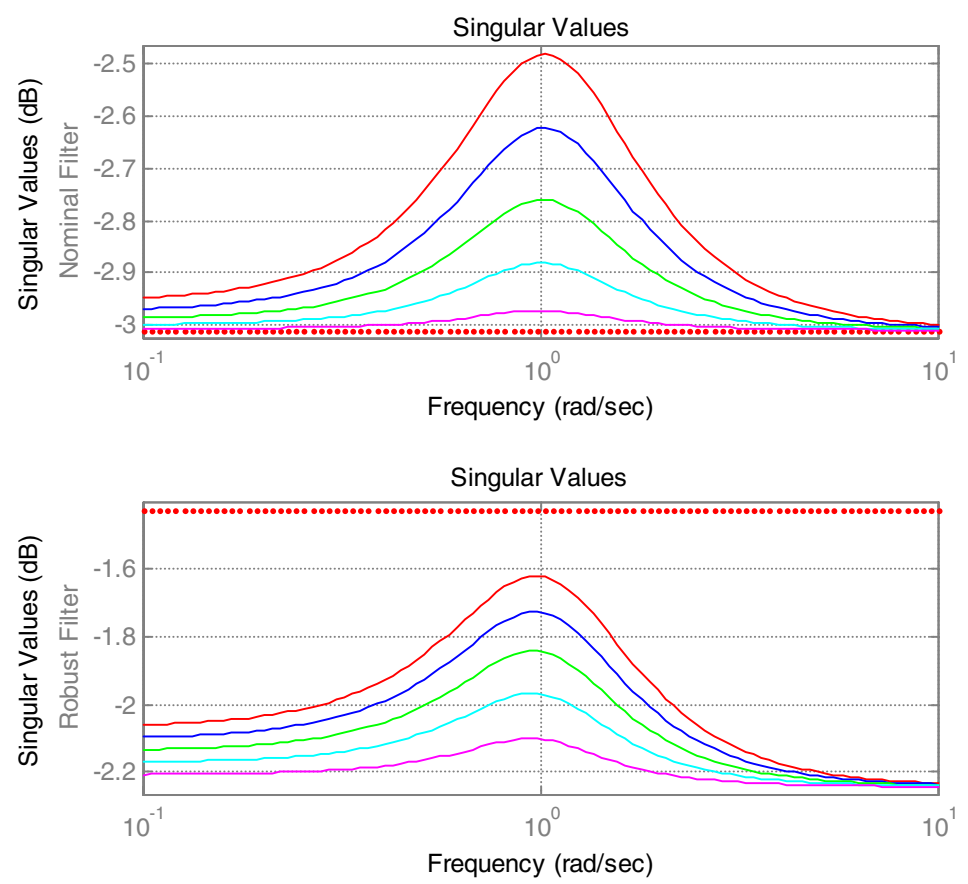

Figure 7. Nominal (above) and robust (below) FDI filter design results.

when the design does not take into account the uncertainty is indeed unbounded. For the robust design, the $\gamma$ value in decibels for the uncertain case now appears consistently in the top of the graph, representing the limit for the singular value plots. However, it may be seen that the nominal design curves are below the respective robust curve, showing a better performance for the given constant uncertainties. This is an apparent contradiction, found also in the other presented designs. It may be explained based on the difference in the $\gamma$ value for the two designs and on the small sensitivity of the used plant related to the uncertainties. The robust design is necessarily conservative, and the scaling matrix is introduced in order to reduce this factor, but the proposed iterative approach cannot guarantee the optimal global solution. However, the lack of an upper bound for the nominal design renders it unreliable, unless an accurate mathematical model of the plant is known.

In Figure 8, the filter designs including the disturbance weight function are presented. The nominal $\gamma$ value is the same 0.7071 and the robust one is 0.8432 , just a little less than the value found before. However, it may be seen that the performance of both the designs are better than in the previous cases, but some of the nominal curves still cross the horizontal bound line. This confirms that the nominal design does not present a reliable upper limit. Again, the robust plots are always below the optimal $\gamma$ value. The main difference, seen in both designs, is the low-pass characteristic in all curves, caused by the introduction of the weight function.

Considering the inclusion of the reference model for the nominal and robust designs, the respective singular value plots may be seen in Figure 9. The $\gamma$ values are, respectively, 0.7071 and 0.8477 . The nominal design curves present the low-pass filter effect, but the high-frequency 

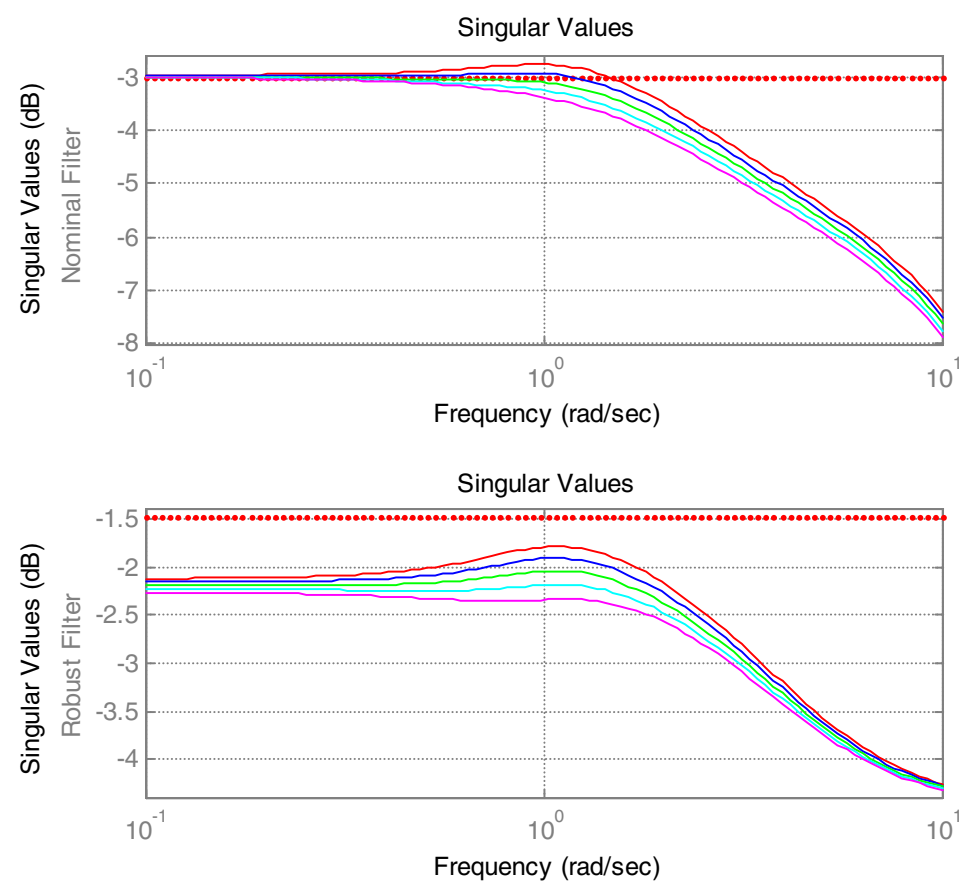

Figure 8. Nominal and robust FDI filter design results, including the disturbance weight function.

rejection is better now compared with the design with the weighting function. Again, some curves are above the respective $\gamma$ value in the low-frequency region. For the robust case, the results are similar to the previous cases. All curves are below the limit, but the high continuous roll-off in the high-frequency region implies a better noise rejection.

\section{CONCLUSIONS}

An $H_{\infty}$ filter design for FDI, based on the estimation of the input fault vector, was presented for the nominal and the uncertain representation of the plant, using an LMI-based approach. Two different sets of LMIs were obtained for each case, whose solution, if the problem is feasible, represents the parameterization of all $H_{\infty}$ FDI filters. For the full-order problem, the filter design constraints are convex, but for the reduced-order filter, an additional rank constraint results in a non-convex problem. An alternating projections algorithm for this case was presented. The robust filtering formulation includes a scaling matrix to reduce the conservatism of this design. For this case, an iterative algorithm is proposed that alternates between the solution of the filter and the scaling parameters, but no global optimum convergence of the algorithm is guaranteed. The formulations were used in a numerical simulated example with several different configurations showing that including a reference model to filter the fault signals improves the performance. However, even including the scaling matrix, the results for the examples are still conservative. The comparison 

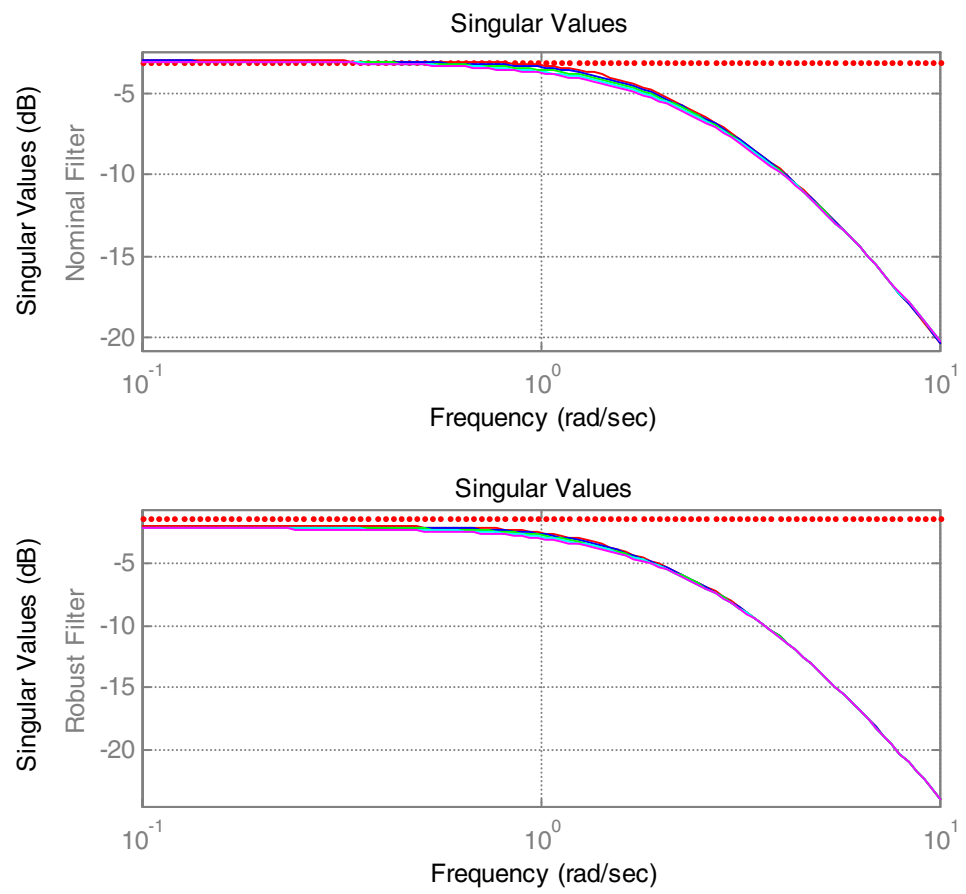

Figure 9. Nominal and robust FDI filter design results, including the reference model.

between the nominal and robust design results, for all examined cases, demonstrates the necessity of the robust design to guarantee a reliable upper bound of the error transfer functions.

\section{APPENDIX A}

\section{A.1. Proof of Theorem 1}

Considering the closed-loop system $F_{l}(S, F)$ from the LFT scheme of Figure 3, and the definitions in (15) and (16), and that the orthogonal complement of $\Gamma$ is

$$
\Gamma^{\perp}=\left[\begin{array}{cc}
{\left[\begin{array}{cc}
B \\
H
\end{array}\right]^{\perp}} & 0 \\
0 & I
\end{array}\right]\left[\begin{array}{ccc}
P^{-1} & 0 & 0 \\
0 & 0 & I \\
0 & I & 0
\end{array}\right]
$$

and defining

$$
\alpha=\left[\begin{array}{l}
B \\
H
\end{array}\right]^{\perp}=\left[\begin{array}{lll}
I & 0 & 0
\end{array}\right], \quad \psi=\left[\begin{array}{cc}
A_{0} P^{-1}+P^{-1} A_{0}^{\mathrm{T}} & 0 \\
0 & -I
\end{array}\right] \quad \text { and } \quad \delta=\left[\begin{array}{c}
B_{0} \\
D_{0}
\end{array}\right]
$$


condition (10) of Lemma 2 leads to the inequality

$$
\alpha \psi \alpha^{\mathrm{T}}+\frac{1}{\gamma^{2}} \alpha \delta \delta^{\mathrm{T}} \alpha^{\mathrm{T}}<0
$$

Partitioning $P$ as

$$
P^{-1}=\left[\begin{array}{cc}
Z & Z_{12} \\
Z_{12}^{\mathrm{T}} & Z_{22}
\end{array}\right]
$$

making $X=Z^{-1}$, and substituting for the LFT matrices in (15), it yields the following inequality:

$$
X A_{\mathrm{s}}+A_{\mathrm{s}}^{\mathrm{T}} X+\frac{1}{\gamma^{2}} X B_{w} B_{w}^{\mathrm{T}} X<0
$$

which corresponds to condition (12) of Theorem 1.

For condition (11) of Lemma 2, considering that

$$
\Lambda^{\mathrm{T} \perp}=\left[\begin{array}{cc}
{\left[\begin{array}{cc}
M^{\mathrm{T}} \\
E^{\mathrm{T}}
\end{array}\right]^{\perp}} & 0 \\
0 & I
\end{array}\right]
$$

and the following definitions

$$
\phi=\left[\begin{array}{c}
M^{\mathrm{T}} \\
E^{\mathrm{T}}
\end{array}\right]^{\perp}, \quad \psi=\left[\begin{array}{cc}
P A_{0}+A_{0}^{\mathrm{T}} P & P B_{0} \\
B_{0}^{\mathrm{T}} P & -\gamma^{2} I
\end{array}\right] \quad \text { and } \delta=\left[\begin{array}{ll}
0 & D_{0}
\end{array}\right]
$$

it can be easily shown that it becomes

$$
\phi \psi \phi^{\mathrm{T}}+\phi \delta^{\mathrm{T}} \delta \phi^{\mathrm{T}}<0
$$

Substituting, it yields

$$
\left[\begin{array}{c}
M^{\mathrm{T}} \\
E^{\mathrm{T}}
\end{array}\right]^{\perp}\left(\left[\begin{array}{cc}
P A_{0}+A_{0}^{\mathrm{T}} P & P B_{0} \\
B_{0}^{\mathrm{T}} P & -\gamma^{2} I
\end{array}\right]+\left[\begin{array}{c}
0 \\
D_{0}^{\mathrm{T}}
\end{array}\right]\left[\begin{array}{ll}
0 & D_{0}
\end{array}\right]\right)\left[\begin{array}{c}
M^{\mathrm{T}} \\
E^{\mathrm{T}}
\end{array}\right]^{\perp \mathrm{T}}<0
$$

Partitioning $P$ as

$$
P=\left[\begin{array}{cc}
Y & Y_{12} \\
Y_{12}^{\mathrm{T}} & Y_{22}
\end{array}\right]
$$

and considering that

$$
\phi=\left[\begin{array}{c}
M^{\mathrm{T}} \\
E^{\mathrm{T}}
\end{array}\right]^{\perp}=\left[\left[\begin{array}{c}
C_{y}^{\mathrm{T}} \\
D_{y w}^{\mathrm{T}}
\end{array}\right]^{\perp} \quad 0\right]\left[\begin{array}{lll}
I & 0 & 0 \\
0 & 0 & I \\
0 & I & 0
\end{array}\right]
$$

inequality (13) results after some simple algebraic manipulations. 


\section{APPENDIX B}

\section{B.1. Proof of Theorem 4}

Considering the closed-loop system $F_{l}\left(S_{u}, F\right)$, from the LFT scheme of Figure 6, the definitions in (27) and (28), and that

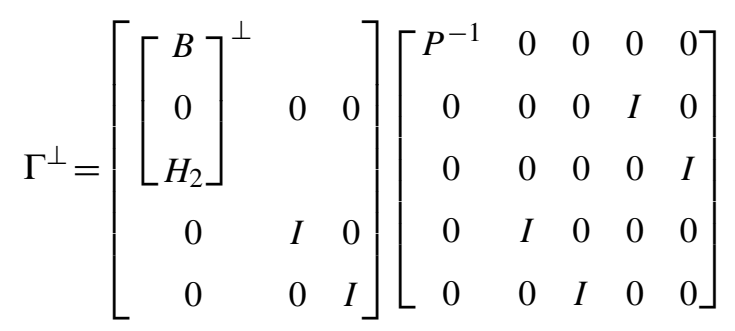

and defining

$$
\begin{aligned}
\alpha & =\left[\begin{array}{c}
B \\
0 \\
H_{2}
\end{array}\right]^{\perp}, \quad \psi=\left[\begin{array}{ccc}
A P^{-1}+P^{-1} A^{\mathrm{T}} & P^{-1} C_{1}^{\mathrm{T}} & P^{-1} C_{2}^{\mathrm{T}} \\
C_{1} P^{-1} & -S^{-1} & 0 \\
C_{1} P^{-1} & 0 & -I
\end{array}\right] \\
\delta_{1} & =\left[\begin{array}{c}
B_{01} \\
D_{011} \\
0
\end{array}\right] \text { and } \delta_{2}=\left[\begin{array}{c}
B_{02} \\
D_{012} \\
D_{022}
\end{array}\right]
\end{aligned}
$$

condition (10) of Lemma 2 yields the inequality

$$
\left[\begin{array}{ccc}
\alpha \psi \alpha^{\mathrm{T}} & \alpha \delta_{1} & \alpha \delta_{2} \\
\delta_{1}^{\mathrm{T}} \alpha^{\mathrm{T}} & -S & 0 \\
\delta_{2}^{\mathrm{T}} \alpha^{\mathrm{T}} & 0 & -\gamma^{2} I
\end{array}\right]<0
$$

Applying the Schur complement formula, it becomes

$$
\alpha \psi \alpha^{\mathrm{T}}+\alpha \delta_{1} S^{-1} \delta_{1}^{\mathrm{T}} \alpha^{\mathrm{T}}+\frac{1}{\gamma^{2}} \alpha \delta_{2} \delta_{2}^{\mathrm{T}} \alpha^{\mathrm{T}}<0
$$

Partitioning $P$ as $P^{-1}=\left(1 / \gamma^{2}\right)\left[\begin{array}{cc}X & X_{12}^{\mathrm{T}} \\ X_{12} & X_{22}\end{array}\right]$, substituting for the LFT matrices in (28), and considering that

$$
\alpha=\left[\begin{array}{ll}
0 & 0 \\
0 & I \\
0 & 0 \\
I & 0
\end{array}\right]^{\perp}=\left[\begin{array}{llll}
I & 0 & 0 & 0 \\
0 & 0 & I & 0
\end{array}\right]
$$

substitution of these terms in (B1) multiplied by $\gamma^{2}$ leads easily to condition (23) of Theorem 4. 
For the second part of Theorem 3, considering that

$$
\Lambda^{\mathrm{T} \perp}=\left[\begin{array}{cccc}
{\left[\begin{array}{c}
M \\
E_{1}^{\mathrm{T}} \\
E_{2}^{\mathrm{T}}
\end{array}\right]^{\perp}} & & & \\
0 & I & 0 \\
0 & & 0 & I
\end{array}\right]
$$

and the definitions

$$
\begin{aligned}
\phi & =\left[\begin{array}{c}
M \\
E_{1}^{\mathrm{T}} \\
E_{2}^{\mathrm{T}}
\end{array}\right]^{\perp}, \quad \psi=\left[\begin{array}{ccc}
P A+A^{\mathrm{T}} P & P B_{01} & P B_{02} \\
B_{01}^{\mathrm{T}} P & -S & 0 \\
B_{02}^{\mathrm{T}} P & 0 & -\gamma^{2} I
\end{array}\right] \\
\delta_{1} & =\left[\begin{array}{lll}
C_{1} & D_{011} & D_{012}
\end{array}\right] \text { and } \delta_{2}=\left[\begin{array}{lll}
C_{2} & 0 & D_{022}
\end{array}\right]
\end{aligned}
$$

it can be easily shown that condition (11) of Lemma 2 becomes

$$
\left[\begin{array}{ccc}
\phi \psi \phi^{\mathrm{T}} & \phi \delta_{1}^{\mathrm{T}} & \phi \delta_{2}^{\mathrm{T}} \\
\delta_{1} \phi^{\mathrm{T}} & -S^{-1} & 0 \\
\delta_{2} \phi^{\mathrm{T}} & 0 & -I
\end{array}\right]<0
$$

Applying the Schur complement formula, it leads to the following inequality:

$$
\phi \psi \phi^{\mathrm{T}}+\phi \delta_{1}^{\mathrm{T}} S \delta_{1} \phi^{\mathrm{T}}+\phi \delta_{2}^{\mathrm{T}} \delta_{2} \phi^{\mathrm{T}}<0
$$

Partitioning $P$ as $P=\left[\begin{array}{cc}Y & Y_{12} \\ Y_{12}^{\mathrm{T}} & Y_{22}\end{array}\right]$ and substituting the respective matrices in (28), it leads to inequality (24).

\section{ACKNOWLEDGEMENTS}

The first author is thankful to FAPESP in Brazil for the financial support during his sabbatical visit at the University of Houston.

\section{REFERENCES}

1. Beard RV. Failure accommodation in linear systems through self-reorganisation. Ph.D. Dissertation, MIT, Cambridge, MA, 1971.

2. Jones HL. Failure detection in linear systems. Ph.D. Thesis, Department of Aeronautics and Astronautics, MIT, Cambridge, U.S.A., 1973.

3. Willsky AS. A survey of design methods for failure detection in dynamic systems. Automatica 1976; 12:601-611.

4. Frank PM. Fault diagnosis in dynamic systems using analytic and knowledge-based redundancy-a survey and some new results. Automatica 1990; 26:459-474.

5. Patton RJ. Robust model-based fault diagnosis: the state of the art. IFAC, Espoo, Finland, 1994; 1-24.

6. Frank PM, Ding X. Survey of robust residual generation and evaluation methods in observer-based fault detection systems. Journal of Process Control 1997; 7(6):403-424.

7. Patton RJ, Frank PM, Clark RN (eds). Fault Diagnosis in Dynamic Systems: Theory and Applications. PrenticeHall: Englewood Cliffs, NJ, 1989. 
8. Chen J, Patton RJ. Robust Model-based Fault Diagnosis for Dynamic Systems. Kluwer Academic Publishers: Boston, U.S.A., 1999.

9. Qiu Z, Gertler J. Robust FDI systems and $H_{\infty}$ optimization. Proceedings of the 32nd IEEE Conference on Decision and Control, San Antonio, U.S.A., 1993; 1710-1715.

10. Frank PM, Ding X. Frequency domain approach to optimally robust residual generation and evaluation for model-based fault diagnosis. Automatica 1994; 30(5):589-804.

11. Douglas RK, Speyer JL. Robust fault detection filter design. Journal of Guidance, Control and Dynamics 1996; 19(1):214-218.

12. Edelmayer A, Bokor J, Keviczky L. Robust detection filter design for linear systems: comparison of two approaches. Proceedings of 13th IFAC World Congress, 7f-01(6), San Francisco, CA, U.S.A., 1996; 37-42.

13. Chen J, Patton RJ. $H_{\infty}$ formulation and solution for robust fault diagnosis. Proceedings of the 14th IFAC World Congress, Beijing, China, 1999; 127-132.

14. Duan GR, Howe D, Patton RJ. Robust fault detection in descriptor linear systems via generalised unknown input observers. Proceedings of the 14th IFAC World Congress, Beijing, China, 1999; 43-48.

15. Hou M, Patton RJ. An LMI approach to $H_{-} / H_{\infty}$ fault detection observers. UKACC International Conference on Control. IEE: London, 1996; 305-310. Conference Publication No. 427.

16. Rambeaux F, Hamelin F, Sauter D. Robust residual generation via LMI. Proceedings of the 14th World Congress of IFAC, Beijing, China, 1999; 241-246.

17. Hamelin F, Sauter D. Robust fault detection in uncertain dynamic systems. Automatica 2000; 36:1747-1754.

18. Casavola A, Famularo D, Franzè G. Robust fault detection of uncertain linear systems via quasi-LMIs. ACC, Portland, Oregon, 2005; 1654-1659.

19. Frisk E, Nielsen L. Robust residual generation for diagnosis including a reference model for residual behavior. Automatica 2006; 42:437-445.

20. Khosrowjerdi MJ, Nikhoukhah R, Safari-Shad N. Fault detection in a mixed $H_{2} / H_{\infty}$ setting. IEEE Transactions on Automatic Control 2005; 50:1063-1068.

21. Zhong M, Ding SX, Bingyong T, Jeinsch T, Sader M. An LMI approach to design robust fault detection observers. Fourth WCICA, Shanghai, China, 2002; 2705-2709.

22. Henry D, Zolghadri A. Design of fault diagnosis filters: a multi-objective approach. Journal of Franklin Institute 2005; 342:421-446.

23. Frisk E, Nielsen L. Robust residual generation for diagnosis including a reference model for residual behaviour. Proceedings of 14th World Congress of IFAC, Beijing, China, 1999; 55-60.

24. Zhong M, Ding SX, Lam J, Wang H. An LMI approach to design robust fault detection filter for uncertain LTI systems. Automatica 2003; 39:543-550.

25. Niemann HH, Stoustrup J. Filter design for failure detection and isolation in the presence of modelling errors and disturbances. Proceedings of the 35th IEEE Conference on Decision and Control, Kobe, Japan, 1996; 1155-1160.

26. Grigoriadis KM, Watson Jr JT. Reduced-order $H_{\infty}$ and $L_{2}-L_{\infty}$ filtering via linear matrix inequalities. IEEE Transactions on Aerospace and Electronic Systems 1997; 33(4).

27. Watson Jr JT, Grigoriadis KM. Optimal unbiased filtering via linear matrix inequalities. Systems and Control Letters 1998; 35:111-118.

28. Li H, Fu M. A linear matrix inequality approach to robust $H_{\infty}$ filtering. IEEE Transactions on Signal Processing 1997; 45(9):2338-2350.

29. Skelton RE, Iwasaki T, Grigoriadis KM. A Unified Algebraic Approach to Linear Control Design. Taylor \& Francis: London, 1998.

30. Grigoriadis KM, Skelton R. Low order control design for LMI problems using alternating projections. Proceedings of the 33rd IEEE Conference on Decision and Control, Orlando, U.S.A., 1996.

31. Grigoriadis KM, Beran E. Alternating projection methods for LMI problems with rank constraints. In Recent Advances in Linear Matrix Inequality Approach to Control, Niculescu S-I, El Ghaoui L (eds). SIAM: Philadelphia, PA, 1999.

32. Nobrega EG, Abdalla MO, Grigoriadis KM. A reference model LMI approach to fault detection and isolation. Proceedings of the IASTED International Conference on Intelligent Systems and Control 2000, Honolulu, U.S.A., 2000; 327-377.

33. Haddad WM, Collins Jr EG, Moser R. Structured singular value controller synthesis using constant D-scales without D-K iterations. International Journal of Control 1996; 63(4):813-830.

34. Packard A, Doyle JC. The complex structured singular value. Automatica 1993; 29:71-109. 